\title{
Debates sobre crianza con apego en medios de comunicación argentinos: mamis famosas y la secta del colecho
}

\section{Debates on attachment parenting in the Argentine media: famous mothers and co-sleeping sect}

\section{Marina Sánchez de Bustamante}

Universidad de Buenos Aires, Buenos Aires, Argentina

marina.sdbagmail.com

\section{Resumen}

Este trabajo analiza debates sobre la crianza con apego que circularon en los medios masivos argentinos sobre la maternidad de mujeres del espectáculo televisivo durante 2014 y 2015. La crianza con apego determina que durante sus primeros años de vida los niños y las niñas necesitan preferiblemente la atención y el cuidado permanente de su madre. Se examina el rol de la cultura de masas en la difusión de ideas y prescripciones sobre el cuidado infantil. Luego, se observan consideraciones en espacios televisivos y artículos periodísticos sobre el cuidado con apego para reflexionar sobre transformaciones actuales de las subjetividades maternas. En conclusión, se interpreta la exhibición de una alerta sobre elecciones de las famosas y la comunidad de madres adherentes, que se ubican mayormente como exóticas e irracionales y -simultáneamente- la expresión de un clima de época en que las mujeres asumen participar en las contiendas de la esfera pública.

Palabras Clave: maternidad, crianza con apego, celebridades, medios de comunicación

\begin{abstract}
This article analyzes discussions about attachment parenting that circulated in the Argentine media about parenting styles among TV celebrities during 2014 y 2015. Attachment parenting implies that during the early years of life infants need permanent care and attention from their mother preferably. It is examined the role of mass culture in the dissemination of ideas and prescriptions on forms of child care. Then, the explanations and opinions offered on television shows and articles about the trend of parenting with attachment are observed to think about on ongoing transformations of maternal subjectivities. In sum, the study identifies the selection of celebrities and follower-mothers, mostly represented as exotic and irrational, and, at the same time, a spirit of the times in which women join and contest their engagement in the public sphere.
\end{abstract}

Keywords: motherhood, attachment parenting, celebrities, mass media 


\section{Introducción}

Entre los delineamientos sociales de la maternidad, el cuidado infantil es una regulación central para los modos en que las mujeres ejercen el rol materno. Los discursos sobre la resolución apropiada de la crianza -es decir, sobre las acciones relativas a aspectos como la alimentación, el descanso, el afecto, la salud y la educación de niñas y niños (Abduca, 2014)- configuran un entramado en el que se expresan disputas entre y al interior de saberes expertos como la pediatría, la psicología y la puericultura. Discursos que, simultáneamente, se exteriorizan en la vida cotidiana de las madres: en sus costumbres de cuidado infantil, en las opiniones y evaluaciones que expresan y reciben sobre sus decisiones.

Esta investigación ${ }^{1}$ describe y analiza consideraciones sobre una corriente de crianza, la crianza con apego, vertidas en textos mediáticos (programas de televisión y artículos periodísticos) durante 2014 y 2015 a partir de la tematización de la maternidad de famosas televisivas. Al producir relatos sobre esas vidas íntimas, los medios masivos vuelven visibles y disponibles diversos modos actuales de ser madre que permiten interrogar imaginarios de época sobre roles e identidades de género, parentesco y filiación, sexualidad y erotismo, parentalidad y cuidado infantil, entre otros tópicos.

Sintéticamente, las ideas fuerza de la crianza con apego sostienen que la función materna pivotea en la naturaleza mamífera, que provee habilidades reflejas para cuidar de los hijos. Que el ejercicio de la maternidad se erige desde la configuración biológica del ser humano como mamífero, cuyas dotes marcan a las mujeres para que se hagan cargo del cuidado infantil en función de rasgos considerados innatos como la capacidad de amamantar y el presunto instinto de cobijo y atención a las necesidades de los bebés, con los que entablan un vínculo de mutua dependencia: una relación de apego.

Al asignarse a las madres las pautas sobre crianza remiten a una cartografía social en la que la adopción de determinadas prácticas designa a las mujeres como mejores o peores madres. El artículo explora estos procesos de configuración y reconfiguración de subjetividades maternas a partir de dos interrogantes: ¿Qué se dice en los medios masivos argentinos sobre la crianza con apego y sobre quienes la practican? ¿Qué voces se jerarquizan para discutir sus implicancias?

El estudio propone un recorrido histórico de los medios de comunicación que repone la estabilización del cuidado infantil en contenidos de la cultura de masas y la disociación contemporánea entre distintas corrientes de crianza. Específicamente, se analiza el cruce entre celebridades y crianza con apego situado en un contexto de demandas y activismos de género en Latinoamérica. Finalmente, se describen y analizan conceptos expresados en algunos debates públicos producidos en línea con la difusión de las prácticas maternales de celebridades televisivas.

\section{Marco teórico}

La línea conceptual de esta propuesta implica una aproximación a operaciones de generización en la producción de textos de las industrias culturales, que reconoce la implicancia de su función de reproducción ideológica y social (Zallo, 1988) aunque sin considerarlas omnipotentes. Parafraseando a Teresa de Lauretis (1996) la maternidad, como el género, no es una propiedad de los cuerpos sino un conjunto de efectos producidos en el despliegue de "tecnologías de género".

Entre esas tecnologías, De Lauretis reconoce procesos de representación mediante la difusión de modelos ya generizados en las formas culturales que se despliegan en cada época. Los discursos -entre ellos los de la industria de la cultura- son configuradores de la experiencia humana y de identidades, poseen un carácter "modelizador respecto a las conductas, sentidos de la buena vida al uso, dimensiones de la experiencia; en definitiva, de todo aquello que aporta a la reproducción del orden social" (Arfuch, 2005). En términos de Silverstone (2004) las industrias culturales son constitutivas de la experiencia colectiva en tanto filtran y forman las realidades cotidianas, entregan palabras para hablar e ideas para expresar y participar de realidades sociales. En este sentido, si la maternidad, como constructo cultural, fue forjándose como una identidad transhistórica y transcultural a partir de la convergencia de discursos y prácticas sociales que la definieron en términos esencialistas y biologicistas (Palomar, 2005), se observa que 
las mediatizaciones que la moldean en la actualidad permite comprenden operaciones de permanencia y perturbación simbólica en torno a esta identidad generizada.

Se siguen los planteamientos de Stuart Hall sobre los procesos dialécticos que estructuran las formas culturales en términos de disputa por la hegemonía (1984), es decir, en la escena situada de luchas que conlleva la contienda por fijar los significados. En tal sentido, observar la producción y circulación de sentidos generizados y generizantes implica atender las relaciones de fuerza y las condiciones históricas en que se desarrollan los modos colectivos de entender y transitar los géneros que se construyen y reconstruyen en contextos específicos. Con relación a estos procesos de contienda semántica y clasificación de sujetos y prácticas, a continuación se aborda el diálogo histórico relativo a maternidad y crianza entre saberes expertos y textos culturales.

\subsection{Crianza, saberes expertos y cul- tura de masas}

Tempranamente, la industria cultural argentina comenzó a divulgar ideas sobre crianza y maternidad apropiadas. A fines del siglo XIX, los primeros títulos fueron fruto del interés pedagógico de moralistas católicos por difundir argumentos de índole moral, filosófica y religiosa (Nari, 2004). Esos textos tuvieron continuidad con publicaciones provenientes de la medicina higienista que se centraron en la divulgación de ideas elementales sobre puericultura (Darré, 2013).

La trama de los discursos sobre crianza y maternidad en textos del mercado editorial argentino, cuyas ideas y valores han sido analizados extensamente en estudios de diversos campos (Abduca, 2014; Borinsky, 2005; Briolotti \& Benítez, 2014; Cosse, 2010; De Paz Trueba, 2011; Rustoyburu, 2012, 2016), da cuenta de un primer período (18901940) en el que la intervención sobre las prácticas maternas fue encabezada por ciencias médicas. En este período la medicina del niño se constituyó como especialidad escindida en dos ramas, orientadas a explicar lo somático en función de lo orgánico (Abduca, 2014): la pediatría, centrada en el abordaje clínico y el seguimiento del crecimien- to, y la puericultura, enfocada en la transmisión de métodos de crianza científicos (Nari, 2004). Tal proceso se situó en el marco de cambios sociales más amplios que implicaron el reconocimiento de la niñez como categoría social y una nueva modelación de la relación niño-adulto (Colangelo, 2012). E implicó también el despliegue del proceso local de maternalización, en el que las mujeres fueron "comprendidas como un bien reproductivo de la nación" (Nari, 2004, p.151), que confluyó en la inherencia semántica entre feminidad y maternidad.

En una coyuntura estatal con metas poblacionistas en la que se evaluaban variables demográficas lcomo la mortalidad infantil y la disminución de la natalidad) articuladas con consideraciones eugenésicas del tejido social y, ligado a esto, en la que se rechazaba la participación de las mujeres en el mercado del trabajo asalariado, la medicina fue la aliada fundamental para intervenir en las prácticas cotidianas. Un escenario en el que, mientras "las mujeres madres fueron presentadas como incompetentes, ignorantes y negligentes, necesitadas de consejos por parte de los profesionales de la salud y de la reproducción" (p.102), las publicaciones dirigidas a las madres agilizaron la transmisión de nuevas pautas culturales de cuidado infantil al recurrir a un lenguaje coloquial.

Se estabilizó, así, lo que Nari identifica como la "crianza científica de los niños" (1995, p. 9) que estableció la figura del médico como instructor y que desconoció la tradición de saberes compartidos, heredados y ejercidos por las mujeres.

Posterior a 1940, se incorporaron progresivamente la psicología y el psicoanálisis con explicaciones sobre aspectos psíquicos, familiares y sociales que se asociaban a necesidades y problemas de los niños (Abduca, 2014). Mientras que las primeras décadas del siglo XX consolidaron la medicalización de la maternidad y la crianza, articuladas a través de políticas estatales de maternalización ${ }^{2}$, las siguientes implicaron una renovación de los saberes que intensificaron las exigencias hacia las madres como cuidadoras de los hijos. Tal viraje sucedió en un contexto de transformaciones políticas y sociales que, en términos sanitarios, se expresaron en el aumento de la natalidad y la disminución de la mortalidad infantil, así como la erradicación de enfermedades que impactaban en la población (tifus, sífilis, paludismo, brucelosis). El modelo asisten- 
cial-protector del Estado durante el gobierno peronista dio cuenta de un avance significativo en la salud colectiva y creó condiciones de posibilidad para el afloramiento de un nuevo paradigma de crianza (Darré, 2013).

El enlace entre industria cultural, maternalización de las mujeres y medicalización de la crianza en Argentina alcanzó un gran esplendor durante los años sesenta y setenta con la eclosión editorial de libros, revistas y colecciones de fascículos especializados, programas radiofónicos y televisivos. En ese período algunos profesionales de la salud -como el pediatra Florencio Escardó, la asistente social y psicóloga Eva Giberti y el pediatra Mario Socolinsky ${ }^{3}$ - fueron referentes pedagógicos de los cuidados infantiles en múltiples textos de medios masivos. La proliferación de estos y otros nombres y de espacios en los que difundían sus consideraciones permite señalar que, desde la década del sesenta, la medicalización de la crianza “se constituiría como un proceso de acción colectiva, vinculado no sólo a los intereses de las corporaciones médicas sino también del mercado y el consumo" (Rustoyburu, 2012, p.84).

Hacia el cierre del siglo XX e inicios del XXI, el modelo de crianza de los productos culturales dirigidos a las madres puede resumirse en lo que Hays (1998) llama "la ideología de la maternidad intensiva”. Esa categoría reúne un conjunto de creencias que naturalizan una perspectiva de crianza centrada en el niño, es decir, que pone el foco en sus necesidades y deseos lya no en las de la nación); cuyos métodos no sólo requieren la guía de expertos sino que también "son más intensivos, más absorbentes desde el punto de vista emocional y económicamente más caros que nunca" (Hays, 1998, p.82).

\subsection{Maternidad intensiva y crianza con apego}

Al aproximarse al mercado editorial español, Jiménez y Roquero (2016) escinden dos repertorios sobre crianza cuya distinción reside en a quién se atribuye la tarea del cuidado y en cómo debe resolverse. Por un lado, identifican un conjunto de voces -"maternidad diversificada y crianza compartida" (p.332)- que considera legítimo delegar el cuidado (en niñeras o instituciones) y que ofrece lineamientos de conductas para la organización y el bienestar del grupo familiar. Por el otro, encuentran un compendio que se opone a las regulaciones tempranas del comportamiento infantil y que cuestiona los criterios del otro conjunto al considerarlos organizados según las necesidades de la vida adulta. Este repertorio - "maternidad intensiva y crianza con apego" (p.334)- afirma que bebés y niños/as pequeños/as requieren atención permanente y que las madres poseen una disposición biológica para dar respuesta a esa necesidad.

La expresión "crianza con apego" nació de la confluencia entre discurso experto, crianza y mercado cultural, cuando un pediatra norteamericano (William Sears) la difundió desde fines de los noventa en sus libros sobre cuidado infantil. Pero fue en los años cincuenta cuando el psiquiatra John Bowlby desarrolló su teoría del apego. Divulgada a partir de un informe elaborado para la Organización Mundial de la Salud, publicado bajo el título Maternal Care and Mental Health (1952), esa teoría sostiene que bebés y niños requieren, para su bienestar, una relación "cálida, íntima y continua con su madre (o permanente sustituto materno), en la cual ambos encuentren satisfacción y placer" (p.11).

Sometido a críticas por la ortodoxia del campo psicológico y revisado posteriormente por la OMS (Briolotti, 2016; Rustoyburu, 2012), marcó igualmente a los saberes sobre salud infantil y posicionó la dimensión afectiva como un aspecto relevante para la medicina familiar psicosomática. Basada en las ideas de Bowlby “contribuyó a naturalizar la supuesta existencia de un lazo afectivo constitutivo entre madre e hijo, con su correlato en la interdependencia para el desarrollo emocional del niño" (Briolotti, 2016, p.434).

En Argentina, la terapeuta Laura Gutman es una de las referentes de la crianza con apego, con una sólida presencia en el mercado editorial. En La maternidad y el encuentro con su propia sombra, afirma que el bebé humano nace prematuramente con respecto a los demás mamíferos, lo que implica que durante los primeros meses de vida requiere condiciones similares a las del vientre materno: comunicación, contacto y atención permanentes (Gutman, 2008). Y "permanente" significa "todo el tiempo". Gutman evalúa que "somos una so- 
ciedad en extremo violenta con nuestra cría" que desoye "los reclamos naturales de los bebés que dependen en forma exclusiva de los cuidados de los adultos" y que "están a merced de nuestras caprichosas ideas modernas" (p.136).

\section{Metodología}

Este artículo se enmarca en el análisis cultural cualitativo desde la tradición de la crítica ideológica (Hall, 1981; Richard, 2009; Thompson, 1991) para indagar en la configuración de sentidos sociales en relación con sus condiciones materiales de producción (Angenot, 2010, Foucault, 1979). Asimismo, el estudio se ubica en el paradigma interpretativo de abordaje abductivo para el acercamiento a los textos culturales (Ginzburg, 1989; Ford, 1994) por ser un dispositivo para la elaboración de hipótesis. Entendemos el análisis cultural como un juego de interpretaciones, una producción de conjeturas a partir de huellas en los discursos que expresan valores e imaginarios posibles en contextos específicos. Dicho análisis supone una lectura zigzagueante que entre y salga de los textos, con la pretensión de reponer una complejidad en constante transformación (Alabarces, 2002).

Seleccionamos aquí un conjunto acotado de materiales extraído de un corpus mayor que conformó un estudio sobre modelos de maternidad que circularon entre 2011 y 2016 en relatos periodísticos sobre madres de la farándula argentina (Sánchez de Bustamante, 2017). El carácter heterogéneo de las fuentes de ese estudio lartículos de revistas de actualidad y espectáculos, de prensa diaria y de portales de noticias sobre chimentos ${ }^{4}$ y espectáculos, informes y entrevistas de programas televisivos de interés general, de chimentos y espectáculos y noticieros) se justificó a partir de la observación de una continuidad cultural en el que la construcción de narrativas y la circulación de información se establece a través de la retroalimentación en la producción, reproducción y ampliación de noticias sobre celebridades de la industria televisiva en las que se tematizan, entre otras cuestiones, aspectos de su vida privada (Justo von Lurzer, 2017). El período de ese trabajo, así como los casos que se categorizaron en distintos modelos (maternidad soltera por elección, maternidad añosa, maternidad apegada, maternidad abyecta y maternidad erotizada), se determinaron luego de la revisión de un fragmento temporal más amplio sobre dos revistas de actualidad argentinas (Caras y Gente). Esa revisión permitió observar la configuración de historias sobre maternidades que tensionaban el arquetipo mediatizado hegemónico de la maternidad (biológica, cis-género, heterosexual, biparental, recatada, jovial, entre otras características) situadas en un contexto de modificaciones legislativas y sociales.

En este trabajo en particular, se analiza un fragmento temporal específico (2014-2015) en el que las elecciones de cuidado infantil de algunas madres famosas alentaron explicaciones y discusiones periodísticas sobre prácticas que se inscriben en la corriente de la crianza con apego. Se considera que ciertas biografías maternales despiertan inquietudes en tanto alguno de los elementos que componen el modelo de madre comprendido socialmente como el más lógico está siendo desafiado. Que esas biografías iluminan, simultáneamente, la intimidad de las estrellas y los cambios en prácticas socio-genéricas. Y por eso los medios se interesan en componerlas. Son redituables porque se centran en vidas que los públicos se interesan en conocer y porque presentan dislocaciones sobre las que es posible debatir y extender la producción de contenidos. En esa producción informativa emergen consideraciones que interesa reponer en tanto son modos de presentar causalidades de experiencias contemporáneas de maternidad.

\section{Crianza con apego, celebridades y activismo}

Observar el cruce de lo biográfico y lo célebre es interrogar un vínculo inexorable. Como rasgo fundante de la cultura de masas la configuración de ese vértice fue una operación regular de la prensa popular desde inicios del siglo XX, aunque el enfoque fue descentrando la constitución de ídolos provenientes de la ciencia, la política y la industria - "ídolos de la producción"- para ampliarse, y concentrarse, en figuras del entretenimiento como actrices, actores, cantantes y deportistas-"ídolos del consumo"- (Lowenthal, 2006, p.130). Como forma específica de la industria cultural, la cultura de la celebridad se señala como una "formación cultural que tiene una función social" (Turner, 2010, 
p.14). Como condición, lo célebre implica "aquello que concita notoriedad pública y en torno de lo que se expresan opiniones y valores" (Justo von Lurzer, 2017: 311.

Las narrativas sobre las madres célebres se diferencian del repertorio pedagógico tradicional en el que textos culturales enseñan a cuidar por medio de una modalidad instruccional sustentada en firmas de profesionales de la salud. Pero aunque las famosas no son expertas, la identificación y el interés que provoca la difusión de sus pronunciamientos y acciones revela el valor social que conlleva su condición de celebridades.

El contexto de enunciación observado refiere a un estado deliberativo (Semán, 2015) y de ampliación de derechos de géneros y sexualidades. La demanda y promulgación de leyes para prevenir, sancionar y erradicar la violencia contra las mujeres, para reconocer la identidad de género autopercibida, para promocionar y garantizar la lactancia materna, para partos asistidos en un marco de respeto, para ampliar los modos de filiación y habilitar diferentes composiciones familiares, describe una época de paulatinas transformaciones. Entre 2012 y 2015, por ejemplo, se promulgaron y reglamentaron varias leyes relativas a derechos de género: la Ley 26.743/12 de Identidad de Género; la Ley 26.862/13 de Reproducción Médicamente Asistida; la Ley $26.873 / 13$ de Promoción y Concientización Pública de la Lactancia Materna; el Código Civil y Comercial de la Nación (Ley 26.994/14) con títulos que incluyen nuevas regulaciones sobre parentesco, filiación y adopción; el Decreto 2035/15 que reglamentó la Ley 25.929/04 de Parto Humanizado.

A partir de este clima de fortalecimiento del movimiento de mujeres ${ }^{5}$ la crianza con apego también se nutre asociándose, a la vez, con otras temáticas como el parto respetado. Y aumenta en significación para madres - de sectores medios y residencia urbana-que se conocen y organizan a partir de su adhesión a esta corriente. Mediante charlas y talleres de instituciones y profesionales o por iniciativas autogestivas de las madres, la apropiación del repertorio de la crianza con apego -o respetuosa, como también se la nombra- da cuenta de un estado colectivo y singular de reflexividad sobre los motivos y beneficios por los cuales adscribir a la totalidad o parte de sus propuestas (Abdala, 2016; Mantilla, 2019). Ese estado colectivo deriva en activismos con líneas de acción como difundir y defender públicamente las ideas y prácticas que sostiene la corriente, en articulación con efectores de salud con nuevos modelos de atención de embarazo, parto y cuidados infantil (Felitti \& Abdala, 2018).

En 2014 la crianza con apego se volvió visible en la agenda de los medios masivos cuando algunas madres famosas se reconocieron adherentes. Esa exteriorización llevó a que se interroguen sus decisiones. La biografización sobre las formas de criar de ciertas madres famosas derivó entre explicaciones y evaluaciones de especialistas y las reivindicaciones de las madres célebres.

Para reflexionar sobre ideas que circularon en torno a esa articulación entre crianza y activismo se recorrerá el modo en que algunos textos mediáticos abordaron la crianza con apego a partir de las elecciones de las famosas.

\section{Mediatizaciones sobre el colecho}

Durante octubre de 2014, Paula Chaves (modelo argentinal contó en un show de gran audiencia que la noche de su casamiento durmió en el lecho nupcial con su hija. La anécdota no fue casual: Marcelo Tinelli, animador del programa, conocía de antemano ciertas elecciones de Chaves respecto a la crianza de su hija; entre ellas el descanso nocturno compartido, también conocido como colecho. En el diálogo, permeado por la comicidad, la modelo refirió, además, a que su hija se alimentaba exclusivamente de su pecho, y ligó sus acciones a "un nuevo método de crianza": la crianza con apego. Confrontándola, el conductor consideró que debía salvaguardar el vínculo conyugal e incorporar otros hábitos: ofrecer biberón y delegar el cuidado. Pero también indagó las implicaciones de la corriente:

Tinelli: Crianza con apego significa que la tenés en el cuarto hasta cuándo, hasta qué momento. Chaves: Hasta que ella quiera.

Tinelli: ¡Ah! ¿Lo decide ella? [risas] ¡Diganmé que es joda! [...].

Chaves.: Los chicos, en algún momento de sus vidas tienen que dormir con sus padres. Somos animales. ¿Cómo duermen los animales? Con su cría cerca. [...] Hay unas camas que se fabri- 
can que son camas de colecho, que es como una extensión de la cama de los padres.

Tinelli: Pero, ¿le van a hacer una cama más grande para que entre Oli? [risas].

Chaves.: Hay un pediatra español que recomienda tirar los colchones en el piso y dormir todos juntos [...].

Tinelli: [...] ¡Te hicieron un lavaje de cerebro! ¿Quién te metió ese verso? ¡Estás en una secta! [risas] Decime cuál. ¡La secta del colecho! La secta del colecho es jodida [...] Alguno debe estar diciendo: "mirá lo que dice este pibe, no se rige por los principios de la naturaleza, lo hace dormir al chico en la habitación de al lado". Mañana voy a averiguar, voy a googlear "la secta del colecho"6.

Luego de esa emisión, la adhesión de Chaves a la crianza con apego derivó -con la referencia explícita al diálogo con Tinelli- en la producción periodística de datos sobre la corriente, realimentada con ejemplos de otras madres famosas. Es decir, a partir de la mediatización de la práctica maternal de una famosa televisiva, varios espacios ofrecieron explicaciones y clasificaciones sobre los criterios y prácticas que componen esta perspectiva del cuidado infantil y plantearon debates en torno a los beneficios y perjuicios de su adopción.

“Las famosas que siguen el colecho, una práctica que trae polémica", tituló una nota del diario $L a$ Nación ${ }^{7}$. Ese artículo definió a la crianza con apego como "un movimiento que tiene cada día más seguidores" y caracterizó el conjunto de prácticas que resumen sus principios: lactancia prolongada, colecho, contacto corporal permanente y respuesta inmediata al llanto. Además, el artículo incluyó adhesiones enfáticas de otras madres televisivas.

Para describir las prácticas de la crianza con apego y la elección de esa modalidad, los medios masivos anudaron el colecho a celebridades enunciándolo como una "polémica mediática" ${ }^{8}$. Pero para evaluar las implicancias del crecimiento de la corriente acudieron al discurso experto: profesionales de la salud dedicados a la divulgación de saberes sobre crianza.

El magazine televisivo $A M$, por ejemplo, presentó el colecho como una controversia con imágenes de madres famosas y la siguiente locución:
Tiempo atrás, que los padres durmieran en la misma cama que sus hijos estaba visto como algo perjudicial para los niños. Hoy se llama colecho y es la palabra de moda en la crianza de los bebés. Esta nueva forma de crianza natural se fue imponiendo poco a poco en la farándula. La modelo Pampita Ardohain escribió en su Twitter: "Viva el colecho" y compartió una imagen que la muestra durmiendo en una cama grande rodeada de sus tres hijos. Otras famosas que adhieren a la crianza con apego son Carla Conte, Paula Chaves y Maju Lozano, que estaba encantada de dormir junto a su hijo aunque también manifestó que Joaquín ya cumplió tres años y todavía no quiere pasarse a su propia camita. "Margarita va a dormir con nosotros hasta los doce", declaró Griselda Siciliani y se manifestó fanática del colecho. [...] El debate crece $y$, sin lugar a dudas, la crianza natural es una tendencia que pisa fuerte. Incluso ya se fabrican camas especiales para facilitar este cambio cultural. ¿Es positivo que padres e hijos duerman juntos? Colecho, ¿sí o no??

En ese informe, la intervención del especialista invitado, el psicólogo Gervasio Díaz Castelli, fue adversa al descanso nocturno compartido con los hijos:

Es un tema que es bueno abrir, genera mucha controversia, mucho fanatismo y mucha militancia. Y siempre cuando la gente se dogmatiza un poco en general puede producir un poco de daño, ¿no? A mí lo que me parece importante es el argumento de la gente que habla del colecho. La cuestión de que es cultural, que en Arabia, que en China. Lo cultural a veces puede producir daño. [...] Yo escribí “El colecho y el niño bonsái" porque planteo que hay ciertas prácticas de los padres que van mutilando el crecimiento de los pibes. Falta cierta sustancia. La teoría del apego, todos repetimos... Pero me parece que es importante tomarnos el trabajo para entender que hay ciertas cosas que tenemos que pensarlas un poquito mejor. La cama dentro de la estructura familiar no es lo mismo que la silla, es una cuestión muy simbólica ${ }^{10}$.

Se dijo en ese programa que el colecho es una moda impulsada por el fanatismo y el activismo y se invi- 
tó a "pensar mejor" por considerar que no obedece a argumentos válidos sino a dogmas arbitrarios. Se reconoció, por otra parte, la dimensión cultural como un elemento organizador de las prácticas de crianza, que se transforman en el acontecer espacio-temporal. Pero esto no abonó una mirada antropológica sino ético-moral: lo cultural no justifica comportamientos perjudiciales y el colecho -como emergente de nuevos modos de cuidado-se evaluó como una acción que produce daños porque, por ejemplo, desoye límites socio-sexuales como el valor simbólico del lecho conyugal.

Otro espacio televisivo (Argentina para armar) dedicó una emisión a debatir sobre "dos tendencias del siglo XXI":

la primera, que decía que a los chicos hay que dejarlos llorar, dejarlos en el cuarto, que se vayan adaptando a este mundo desde que son bebés, y esta otra, que está tan de moda, que habla de una crianza con apego, donde estamos mucho más pendientes de los chicos, más pegados a los chicos $[. . .]^{11}$.

Para la ejemplificación se convocó justamente a Paula Chaves y para la discusión, entre otras mujeres, a dos terapeutas con posturas contrapuestas cuyo desacuerdo giró en torno a los condicionamientos culturales.

Una de ellas subrayó que las madres desean cumplir con otros roles sociales y actividades, que muchas no eligen el colecho sino que no logran otra forma de sueño continuo y afirmó que lo problemático es que la práctica se explique como una necesidad emocional materna, es decir como una nueva norma cultural:

una mamá que necesita trabajar, que quiere ir al gimnasio, que quiere dormir tranquila en su cama, que quiere dormir abrazada a su pareja, $y$, sin embargo, le llega desde afuera que debería sentir otra cosa, que debería tener ganas de estar con su hijo todo el tiempo, que debería claudicar su parte de ser mujer por ser mamá [... $]^{12}$.

La otra especialista enfatizó que los niños nacen dependientes de los cuidados maternos y que sus necesidades se vinculan a instintos propios de la especie mamífera humana:
Nacemos dependientes de los cuidados maternos, prácticamente sin poder hacer nada, dependemos de la protección de mamá o de la persona maternante. Del cariño, del cobijo, del cuerpo. ¿Qué es lo que hacemos cómo niños humanos? Pedimos, buscamos, necesitamos el cobijo. Esto es diseño original, no es cultura. [...] Acá, más allá de lo que nosotros decidimos, es si nos damos la posibilidad los adultos, y las madres especialmente, de volver a conectar con la niña o el niño que hemos sido gracias al niño que hemos tenido ${ }^{13}$.

De esta forma, ideas contrarias sobre la crianza -una donde los hijos deben adaptarse al ritmo del entorno cultural y otra en la que la cultura no debe interferir con el ritmo de la naturaleza- fueron esbozadas por dos voces del discurso experto y del mercado cultural que se dirige a las mujeres madres. Una de esas voces consideró que las madres deben atender deseos y responsabilidades que trascienden el rol materno. La otra voz propuso escuchar al mundo infantil concebido como no permeado por la cultura, y reivindicó la dimensión biológica humana como "diseño original".

Posteriormente a este período mediático de interés en la crianza con apego a partir de vivencias de las madres famosas, la referencia a la corriente ya no pudo ser soslayada en la producción de contenidos. Cuando eso ocurrió, la omisión tuvo que ser subsanada. Por ejemplo, en 2017 el programa Morfi, todos a la mesa invitó a un pediatra que objetó el colecho y ofreció pautas para que los bebés "aprendan a dormir"14. El descontento que se expresó sobre esa intervención en las redes sociales, con críticas por presentar una sola mirada en torno al descanso de los lactantes, resultó en un inmediato nuevo envío con consideraciones del mismo pediatra y con las opiniones de una puericultora afín a la crianza con apego ${ }^{15}$.

\section{Conclusiones}

En el siglo XXI, el modelo de la maternidad intensiva sobrevivió a transformaciones culturales como la presencia masiva de las mujeres en el mercado del trabajo asalariado, las diversas formas de hacer familia y cierta gradual mutación del rol paterno. Esto implica que las madres continúan po- 
sicionadas como las mejores cuidadoras posibles y como quienes deben asumir las tareas de cuidado. Pero mientras la vigencia de la feminización del cuidado se mantiene como "uno de los núcleos críticos de la construcción social del género" (Faur, 2014, p.14), las perspectivas sobre las formas de crianza complejizan la escena discursiva al presentar argumentos disímiles sobre quién debe asumir la tarea y sobre cómo debe realizarse.

Al situar la dimensión biológica humana como la que orienta las demandas infantiles y las respuestas adecuadas a esas necesidades, la crianza con apego abrió un diálogo crítico con otras pautas que aconsejan regular los comportamientos de los lactantes. Como mencionamos, la irrupción de tal perspectiva se gestó en la confluencia de saberes expertos y la cultura de masas con la prédica de los libros y de las charlas de los especialistas afines. En los grupos de lactancia, crianza y tribus de madres presenciales y virtuales, la difusión de las ideas creció y fue incorporando distintas aristas. Asimismo, la crianza con apego se consolidó no sólo como una opción de cuidado sino como una oportunidad de organización colectiva: una causa a divulgar, que cobró mayor difusión aún al ser tematizada en narrativas periodísticas sobre las madres televisivas.

Con los pronunciamientos de adhesión de algunas madres famosas, la crianza con apego fue presentada a los públicos masivos como "polémica". El colecho, investido en su sinécdoque, en ocasiones fue impugnado con distintas caracterizaciones: por ser una moda, por impulsarse desde el fanatismo y la militancia, por alterar límites de la moral socio-sexual, por imponerse como nueva norma, por implicar un riesgo para los lactantes. Para algunos divulgadores en salud el descanso compartido transgrede fronteras de la moral sexual (concebidas necesarias para proteger a los infantes). Pero para otros la transgresión ética se expresa en desatender las demandas de bebés-cachorros que requieren por instinto animal ese cobijo; para estos, la medicina estabilizó un conjunto arbitrario de acciones distante de los impulsos mamíferos; para aquellos, los nuevos principios adoctrinan con otras normas más perjudiciales.

Por otra parte, las referencias mediáticas al crecimiento de esta corriente exhiben cierta alerta sobre elecciones que se ubican no sólo como exóticas sino también como poco obedientes a ejercer el rol materno bajo la histórica tutela de la medicina, e incluso como desconsideradas respecto al vínculo conyugal. En ese ingreso a la escena mediática de voces que argumentan sus elecciones de crianza se reproduce la naturalización de las tareas de cuidado como potestad de las madres, aunque en los intersticios de esas discusiones también se cuela el clima de época respecto a la puja de las mujeres por el reconocimiento de su protagonismo en la construcción de lo social. Porque las discusiones que emergen desde los grupos y adherentes a la crianza con apego no pueden escindirse de otras, como las impulsadas por el movimiento del parto humanizado que también aumentó su visibilidad.

Esas experiencias comparten, entre otros aspectos, lenguajes anclados en la biología y -como deriva- en esencialismos de género; simultáneamente, sostienen ideas relativas a la autonomía y a la crítica social asociadas a la militancia por los derechos de las mujeres (Felitti \& Abdala, 2018). Es decir, entre la diversidad de demandas y posicionamientos, estos activismos valoran y reclaman el respeto por las decisiones de las madres y resisten el anclaje en la medicina como único discurso válido. En esa resistencia se expresan desacuerdos que, por un lado, se sustentan de fundamentos conceptuales; por el otro lado, se discute cómo los especialistas de la salud ejercen la profesión: la nula escucha y contemplación de las emociones, los conocimientos y las convicciones de las madres por la aplicación de protocolos que excluyen sus deseos y sus creencias.

Si el proceso de maternalización esculpió a las madres como sujetos negligentes, ignorantes, carentes de saberes válidos -y aún el discurso de la maternidad continúa con las lógicas de un dispositivo pedagógico exigente y opresivo- las madres de hoy distan de las de la primera mitad del siglo XX. Muchas son mujeres protagonistas del proceso de avance de las luchas por la igualdad de los géneros que apelan a distintos, y a veces contradictorios, marcos teóricos para reivindicar demandas que conciben más justas para la vida propia y compartida. Entre esas demandas, la problematización acerca de los límites del sistema médico a la autonomía del cuerpo y a las decisiones de las mujeres es uno de los umbrales en la escena contemporánea de cambios en las subjetividades de género. Porque para tomar la voz, las madres (célebres y 
anónimas) se han comprendido a sí mismas como interlocutoras válidas que buscan negociar con otros agentes las prácticas que las involucran y que definen los modos de organizar la vida social.

\section{Notas}

1 Una versión preliminar de este trabajo fue presentada en el Primer Congreso Latinoamericano de Estudios de Género y Cuidado "Miradas latinoamericanas al cuidado", desarrollado del 6 al 8 de noviembre de 2018 en Montevideo, Uruguay. Tal presentación se inscribió en el marco del Proyecto de Investigación Orientada (20152018): “La politización del espectáculo: producción, textos y recepción de los programas de espectáculos televisivos" (Res. N03333) financiado por el Consejo Nacional de Investigaciones Científicas y Técnicas y la Defensoría del Público de Servicios de Comunicación Audiovisual.

2 En relación a las "políticas de maternidad" o las madres como objeto de políticas públicas, Nari (2004) analiza la discusión y sanción de leyes ligadas a la participación de las mujeres en el mercado laboral, al infanticidio, el abandono, el aborto y la tutela de los hijos.

3 Florencio Escardó escribió columnas en Mundo Argentino, Hijo Mío...! Madre y Niño, Viva Cien Años, El Hogar, Vea y Lea, Claudia, Para Ti, Vosotras y Primera Plana. Publicó Anatomía de Familia (1955), Sexología de la Familia (1961) y Mis padres y yo. Nueva puericultura para mamás (1968) y dirigió dos revistas maternales: Mi hijo y yo y Maminia. Eva Giberti escribió columnas en La Razón, Nuestros Hijos, Claudia, Vosotras y Damas y Damitas que luego fueron compiladas en la colección Escuela para Padres (1961). Mario Socolinsky condujo desde fines de los setenta el programa televisivo La Salud de Nuestros Hijos, ciclo emitido hasta 2003.

4 En Argentina, la expresión “programa o revista de chimentos" refiere a productos de la industria audiovisual y gráfica que tematizan la vida íntima de personas públicas ligadas, principalmente, al espectáculo. Para ampliar sobre los aspectos más relevantes y el surgimiento del género mediático "chimentos" en Argentina puede consultarse el trabajo de Alina Mazaferro (2014) sobre la "cultura de las celebridades" y el de Carolina Justo von Lurzer (2017) sobre las funciones sociales de los programas televisivos de espectáculos y chimentos.

5 En Argentina, a partir de 2015, el movimiento de mujeres creció en visibilidad pública con eventos impulsados por reclamos en torno a la violencia y las desigualdades que padecen las mujeres, las lesbianas, las travestis y las personas transgénero. Las manifestaciones que acontecieron con el nacimiento del colectivo Ni Una Menos llas marchas contra los femicidios y los paros de las mujeres), articuladas con otras organizaciones ligadas a demandas feministas, nutrieron y amplificaron los debates que se tradujeron gradualmente, por ejemplo, en el mayor tratamiento mediático sobre problemáticas de género lla violencia física y simbólica, las desigualdades en el trabajo productivo y reproductivo y las políticas vinculadas a la salud sexual y reproductival.

6 Eltrece (14/10/2014): Showmatch 2014 - Tinelli se indignó con un dato del casamiento de Paula y Peter [video]. Recuperado de https://www.youtube.com/ watch?v=8Bd8gvp6Vrs

7 La Nación (10/11/2014): Las famosas que siguen el colecho, una práctica que trae polémica [online]. Recuperado de https://www.lanacion.com.ar/ espectaculos/las-famosas-que-siguen-el-colecho-unapractica-que-trae-polemica-nid1740257

8 Clarín (7/10/2014): ¿Es bueno dormir con los hijos? El colecho, de la TV a tu casa [online]. Recuperado de https://www.clarin.com/hijos/colecho-hijos-bebesninos-padres-dormir-habitacion-cuna-misma-camacrecer-crecimiento-etapas_0_B16SN9YPXI.html

9 Telefe (28/1/2015): Colecho: domir con tu bebé, ¿sí o no? - AM [video]. Recuperado de https://www.youtube. com/watch?v=zcjrGx8LMTY

10 Telefe (28/1/2015): ¿Es bueno dormir con tu bebé? AM [video]. Recuperado de https://www.youtube.com/ watch?v=l-4fnwQT9ag

11 Milii ramos (9/11/2014): Paula Chaves en Argentina para armar 1/2 \#TN [video]. Recuperado de https://www. youtube.com/watch?v=odl-rqJe8AQ

12 Ídem

13 Ídem

14 Telefe (26/01/2017): ¿Cómo hacer que tu bebé duerma? - Morfi [video]. Recuperado de https://www. youtube.com/watch?v=QHPbhCvHC5s

15 Telefe (31/01/2017): Debates de mamás: Colecho si o no? - Morfi [video]. Recuperado de https://www.youtube. com/watch?v=9sF_NPNvzpQ 


\section{Referencias}

Abdala, L. (2016). Exploración sociológica de los significados y prácticas de la maternidad en mujeres que reivindican "partos humanizados" y "crianzas naturales". Tesis de maestría, Facultad de Humanidades y Ciencias, Universidad Nacional del Litoral, Argentina.

Abduca, L. (Julio, 2014). Representaciones sobre la crianza y la niñez en el transcurso del siglo XX en la Argentina. El pasado en el presente. Trabajo presentado en XI Congreso Argentino de Antropología Social, Rosario.

Alabarces, P. (2002). Fútbol y patria. El fútbol y las narrativas nacionales en la Argentina. Buenos Aires: Prometeo.

Angenot, M. (2010). El discurso social. Buenos Aires: Siglo XXI.

Arfuch, L. (2005). “Problemáticas de la identidad”, en Arfuch Leonor (comp.) Identidades, sujetos y subjetividades (pp.21-43). Buenos Aires: Prometeo Libros.

Bowlby, J. (1952). Maternal Care and Mental Health. Washington: OMS.

Borinsky, M. (2005). 'Todo reside en saber qué es un niño'. Aportes para una historia de la divulgación de las prácticas de crianza en la Argentina". Anuario de Investigación, (13), 117-126. Secretaría de Investigaciones, Facultad de psicología, UBA.

Briolotti, A. (2016). El problema del hospitalismo en la medicina rioplatense y el rol de los saberes psi en la subjetivación de la maternidad y la infancia (1933-1965). Rev. Cienc. Salud, 14 (3), 423-438.

Briolotti, A. \& Benítez, S. (2014): Medicina, higiene mental y saber psi en la construcción de la maternidad y la infancia en la Argentina: un análisis de la revista Madre y Niño (1934-1935). Universitas Psychologica, 13 (5), pp.1709-1719.

Colangelo, M. A. (2012). La crianza en disputa: medicalización del cuidado infantil en la Argentina entre 1890 y 1930. Tesis de Doctorado, Facultad de Ciencias Naturales y Museo, Universidad de La Plata.

Cosse, I. (2010): Pareja, sexualidad y familia en los años sesenta. Una revolución discreta en Buenos Aires. Buenos Aires: Siglo XXI Editores.

Darré, S. (2013). Maternidad y tecnologías de género. Buenos Aires: Ed. Katz.

De Lauretis, T. (1996). Tecnologías del género. Revista Mora, 2, 6-34.

De Paz Trueba, Y. (2011). El discurso de la maternidad moderna y la construcción de la modernidad a través de la prensa. El centro y sur bonaerenses a fines del siglo XIX y principios del XX. Quinto Sol, (15) 2 - Online.

Faur, E. (2014). El cuidado infantil en el siglo XXI. Mujeres malabaristas en una sociedad desigual. Siglo XXI: Buenos Aires. 
Felitti, K. \& Abdala, L. (2018): El parto humanizado en la Argentina: activismos, espiritualidades y derechos, en Sanchez, G. \& Laako, A. (ed.) Parterías de Latinoamérica. Diferentes territorios, mismas batallas (pp.123-152). San Cristóbal de las casas: El Colegio de la Frontera Sur.

Ford, A. (1994). Navegaciones. Comunicación, cultura y crisis. Buenos Aires: Amorrortu editores.

Foucault, M. ([1970] 1979]. La arqueología del saber. México, Siglo XXI Editores.

Ginzburg, C. (1989). "Morelli, Freud y Sherlock Holmes: indicios y método científico”. En Eco, U. y Sbeok, T. (ed.) El signo de los tres (pp.116-163). Barcelona: Lumen.

Gutman, L. (2008). La maternidad y el encuentro con la propia sombra. Paidós: Buenos Aires.

Hall, S. (1981). “La cultura, los medios de comunicación y el efecto ideológico”. En Curran, J. et al (ed.) Sociedad y comunicación de masas (pp.357-392). México: FCE.

Hall, S. (1984): "Notas sobre la deconstrucción de lo popular". En Samuels, R. (ed.) Historia popular y teoría socialista (pp.93-110). Barcelona: Crítica.

Hays, S. (1998). Las contradicciones culturales de la maternidad. Paidós: Barcelona.

Jiménez, C. \& Roquero, E. (2016). “Los discursos expertos sobre crianza y maternidad: aproximación al caso español 1950-2010". Arenal. Revista de Historia de las Mujeres, (23), $321-345$.

Justo von Lurzer, C. (2017). Esto le puede servir a alguien. Demandas de derechos en el espectáculo televisivo contemporáneo en Argentina. Estudos em Comunicação, 25 (1), 23-52.

Lowenthal, L. ([1944] 2006). “The triumph of mass idols". En Marshall, P.D. (ed.) The Celebrity Culture Reader (pp.124-152). New York: Routledege.

Mantilla, M.J. (2019). Cuerpos, niñez y crianza: cartografías corporales de la infancia en el modelo de crianza respetuosa en Argentina. Rev. urug. Antropología y Etnografía [online] (4), 1, 36-50.

Mazaferro, A. (2014) La génesis de la cultura de la celebridad. Televisión y cuerpos de la fama en la Argentina (1958-1974). Tesis doctoral de la Facultad de Ciencias Sociales, Universidad de Buenos Aires, Buenos Aires.

Nari, M. (1995). La educación de la mujer lo acerca de cómo cocinar y cambiar los pañales a su bebé de manera científica). Mora, 1, 31-45.

Nari, M. (2004). Políticas de maternidad y maternalismo político. Buenos Aires, Biblos.

Palomar, C. (2005). Maternidad: historia y cultura. Revista de Estudios de Género La ventana, 22, 35-67. 
Richard, N. (2009). La crítica feminista como modelo de crítica cultural. Debate feminista, $20,(40), 75-85$.

Rustoyburu, C. (2012). Infancia y maternidad en los discursos de la pediatría psicosomática (Buenos Aires, a mediados del siglo XX). Tempos Históricos, (16), 59-86.

Rustoyburu, C. (2016). Maternidad e infancia. Los consejos médicos y psicológicos en la revista Hijo Mío...! Buenos Aires, en la década de 1930. ARENAL, (23) 1, enerojunio, 117-142.

Sánchez de Bustamante, M. (2017). Mamis famosas. Narrativas biográficas de la maternidad en la cultura de masas. Tesis de maestría, Facultad de Ciencias Sociales, Universidad de Buenos Aires.

Semán, P. (2015). El Posporno no es para que te excites. Revista Anfibia, Buenos Aires, julio. http://revistaanfibia.com/ensayo/el-posporno-no-es-para-que-te-excites/

Silverstone, R. (2004). ¿Por qué estudiar los medios? Buenos Aires: Amorrortu.

Thompson, J. (1991). La comunicación masiva y la cultura moderna. Contribución a una teoría crítica de la ideología. Revista Versión. Estudios de comunicación y política, 1, 43-76.

Turner, G. (2010). Approaching celebrity studies. Celebrity Studies, 1 (1), 11-20.

Zallo, R. (1988). Economía de la comunicación y la cultura. Madrid: Akal

- Sobre la autora:

Marina Sánchez de Bustamante es becaria y docente de la Universidad de Buenos Aires. Licenciada en Ciencias de la Comunicación y magister en Comunicación y Cultura de la Facultad de Ciencias Sociales (UBA), realiza el Doctorado en Ciencias Sociales.

- ¿Cómo citar?

Sánchez de Bustamante, M. (2019). Debates sobre crianza con apego en medios de comunicación argentinos: mamis famosas y la secta del colecho. Comunicación y Medios, (40), 156-168. 